Pacific Journal of Mathematics

ARENA REGULARITY SOMETIMES IMPLIES THE RN 


\title{
ARENS REGULARITY SOMETIMES IMPLIES THE RNP
}

\author{
A. ÜLGER
}

Let $A$ be a Banach algebra with a bounded left approximate identity. We denote, respectively, by $\operatorname{wap}(A)$ and $A A^{*}$ the subspaces of $A^{*}$ consisting of the weakly almost periodic functionals and the functionals of the form ${ }_{a} f$, where ${ }_{a} f(x)=f(a x)$. The main results can be summarized as follows:

(a) $\operatorname{wap}(A) \subseteq A A^{*}$ and the equality $\operatorname{wap}(A)=A A^{*}$ holds if $A$ is a right ideal in its second dual.

(b) If $A$ is Arens regular and a right ideal in its second dual then $A^{*}$ has the RNP (Radon-Nikodym Property).

(c) If $A$ is a right ideal in its second dual then $A$ is Arens regular and has the Dunford-Pettis property iff $A^{*}$ has the RNP and the Schur property.

As applications we give very short (and probably new) proofs of several well-known results about topological groups, group algebras and their weakly almost periodic functionals. Our applications also include some new proofs and results about the projective tensor products of Banach spaces and algebras.

1. Introduction. In [2] $\mathrm{R}$. Arens has shown that, given any Banach algebra $A$, there exist two algebra multiplications on the second dual $A^{* *}$ of $A$ extending that of $A$. When these two multiplications coincide on $A^{* *}$, the algebra $A$ is said to be (Arens) regular. Details of the construction of these multiplications can be found in many places, including the book [4], the poineering papers [2], [10] and the survey article [13].

In the papers [21], [42], [53], [54] the geometry of Banach spaces has been exploited to obtain results about Arens regularity of certain Banach algebras. In this note we present some results which in turn show that, for a certain class of Banach algebras $A$, the Arens regularity of $A$ implies the presence of the Radon-Nikodym property (RNP), which is a property that belongs to the geometry of Banach spaces, on the dual space $A^{*}$ of $A$. The results presented in this note apply to a Banach algebra $A$ having a bounded left (or right) approximate identity (in short BLAI) and the main hypothesis is automatically satisfied when the algebra in question is a right (or left) ideal in its second dual $A^{* *}$ equipped with either Arens multiplication. Before stating 
the main results of this note we introduce some notation. Let $A$ be a Banach algebra with a BLAI $\left(e_{\alpha}\right)_{\alpha \in I}$. For $a$ in $A$ and $f$ in $A^{*}$, ${ }_{a} f$ is the functional on $A$ defined by ${ }_{a} f(x)=f(a x)$. By $A A^{*}$ we denote the subspace of $A^{*}$ consisting of the functionals of the form ${ }_{a} f$. For each $\alpha$ in $I, H_{\alpha}$ denotes the subset $H_{\alpha}=\left\{e_{\alpha} f: f \in A^{*}\right.$, $\|f\| \leq 1\}$ of $A^{*}$, and $\operatorname{wap}(A)$ denotes the subspace of $A^{*}$ consisting of the weakly almost periodic functionals on $A$ (defined below). The main results of this note can be summarized as follows:

(a) The set $\operatorname{wap}(A)$ is contained in $A A^{*}$ and the equality (wap $(A)=$ $\left.A A^{*}\right)$ holds if $A$ is a right ideal in its second dual.

(b) If, for each $\alpha$ in $I$, the set $H_{\alpha}$ has the RNP and $A$ is regular then $A^{*}$ has the RNP.

(c) If $A$ is a right ideal in its second dual then $A$ is regular and has the Dunford-Pettis property iff $A^{*}$ has the RNP and the Schur property. The main ingredients of the proofs are Cohen-Hewitt factorization theorems [32; 32.22 and 32.24]. As applications of these results we give very short (and probably new) proofs of several well-known results about topological groups, group algebras and their weakly almost periodic functionals. Applications also include some new proofs of known results and some new results about the injective and projective tensor products of Banach spaces and algebras. Some of the applications are the following:

1. If $G$ is a compact topological group and $A=L^{1}(G)$ is its group algebra then $A$ is regular iff $G$ is finite.

2. If $G$ is a locally compact topological group and $A=L^{1}(G)$ is its group algebra then $A$ is a right ideal in its second dual iff $G$ is compact.

3. If $G$ is any compact topological group then the space $C(G)$ does not contain an isomorphic copy of $l^{\infty}$.

4. If $G$ is a compact topological group then $G$ is extremally disconnected (or $\sigma$-Stonean or an $F$-space) iff $G$ is finite.

5. If $X$ and $Y$ are two reflexive Banach spaces with the a. p. (approximation property) then their projective tensor product $X \hat{\otimes} Y$ has the RNP.

6. If $A$ and $B$ are two regular Banach algebras with BLAI and if, for each $a$ in $A$ and each $b$ in $B$, one of the left multiplication operators $a^{\tau}: A \rightarrow A\left(b^{\tau}: B \rightarrow B\right)$, defined by $a^{\tau}(x)=a x$, is compact and the other is weakly compact then

(i) the projective tensor product algebra $A \hat{\otimes} B$ is a right ideal in its second dual, 
(ii) $A \hat{\otimes} B$ is Arens regular iff every bounded linear operator $u$ : $A \rightarrow B^{*}$ is compact,

(iii) if $A \hat{\otimes} B$ is Arens regular then the space $L\left(A, B^{*}\right)=(A \hat{\otimes} B)^{*}$ has the RNP.

In $\S 2$ we have collected the notation and the basic facts about Arens regularity and RNP. The main results are in $\S 3$ and the applications are in $\S 4$ and $\S 5$.

Professor J. S. Pym has read the first draft of the paper and made several valuable suggestions. In particular the proof of Lemma 3.4 is suggested by him. The author gratefully acknowledges here his debt to J. S. Pym.

2. Notations and preliminaries. Besides some general notation and results we shall need some notation and results about Arens regularity and the RNP.

For any Banach space $X$ (real or complex), we denote by $X^{*}$ its dual and by $X_{1}$ its closed unit ball. The natural duality between $X^{*}$ and $X$ is denoted by $\langle f, x\rangle$. We shall regard $X$ as naturally embedded into $X^{* *}$. If $X$ and $Y$ are two Banach spaces, $L(X, Y)$ (resp. $K(X, Y))$ denotes the space of bounded (resp. compact) linear operators from $X$ into $Y$. The spaces $L(X, X)$ and $K(X, X)$ will be denoted by $L(X)$ and $K(X)$, respectively. These spaces are equipped with their usual operator norms. By $X \ddot{\otimes} Y$ and $X \hat{\otimes} Y$ we denote, respectively, the injective and projective tensor products of $X$ and $Y$ [12, Chapter VIII]. $c_{0}, l^{p}(1 \leq p \leq \infty)$ denote the usual sequence spaces. For any topological (Hausdorff) space $G, C(G)$ denotes the Banach space of bounded continuous functions from $G$ into $\mathbb{R}$ or $\mathbb{C}$ endowed with the supremum norm. For a locally compact space $G$, $C_{0}(G)$ is the subspace of $C(G)$ consisting of the functions vanishing at infinity. Equipped with the pointwise multiplication, the spaces $c_{0}$, $l^{p}, C(G)$ and $C_{0}(G)$ are Banach algebras. If $G$ is a locally compact topological group, by $\operatorname{LUC}(G), \operatorname{RUC}(G)$ and $\operatorname{UC}(G)$ we denote, in order, the subspaces of $C(G)$ consisting of the left (right, and both left and right) uniformly continuous functions on $G$ [3, p. 104]. We remark here that in the treatise of Hewitt and Ross [31, p. 275] the space $\operatorname{LUC}(G)$ is denoted by $C_{r u}(G)$ and the space $\operatorname{RUC}(G)$ by $C_{l u}(G)$. By WAP $(G)$ we denote the subspace of $C(G)$ consisting of the weakly almost periodic functions on $G$ [3, p. 107].

Our basic reference about Banach algebras is the book [4]; for any undefined notion used in the present note we refer the reader to this book. If $A$ is a Banach algebra and $a$ is an element of $A$, by $a^{\tau}: A \rightarrow A$ 
we denote the left multiplication operator defined by $a^{\tau}(x)=a x$. The adjoint $a^{\tau *}: A^{*} \rightarrow A^{*}$ of $a^{\tau}$ is given by $a^{\tau *}(f)=a f$ where $a f$ is the functional on $A$ defined by $a f(x)=f(a x)$. Thus the set $H(a)=\left\{a f: f \in A_{1}^{*}\right\}$ is the image of $A_{1}^{*}$ under $a^{\tau *}$. Hence the set $H(a)$ is weakly compact iff the operator $a^{\tau}$ is weakly compact. Here we remark that the set $H(a)$ is always weak *-closed, convex and bounded. We recall that, for each $a$ in $A$, the set $H(a)$ is weakly compact iff $A$ is a right ideal in its second dual, i.e. $A A^{* *} \subseteq A[13$, p. 318, Lemma 3]. We also note that for the multiplication $(a, f) \rightarrow$ af,$A^{*}$ is a right a $A$-module in the sense of [32, p. 263]. A net $\left(e_{\alpha}\right)_{\alpha \in I}$ in $A$ is said to be a BLAI (bounded left approximate identity) ${ }^{1}$ if, for each $\alpha$ in $I,\left\|e_{\alpha}\right\| \leq 1$ and, for all $a$ in $A,\left\|e_{\alpha} a-\alpha\right\| \rightarrow 0$. BRAI (bounded right approximate identity) and BAI (bounded approximate identity) are defined similarly. A functional $f$ in $A^{*}$ is said to be wap (weakly almost periodic) on $A$ if the set $\left\{a f: a \in A_{1}\right\}$ is relatively weakly compact. This is equivalent to the following "Double Limit Criterion" [26]: for any two sequences $\left(a_{n}\right)$ and $\left(b_{m}\right)$ in $A_{1}$,

$$
\lim _{n} \lim _{m}\left\langle f, a_{n} b_{m}\right\rangle=\lim _{m} \lim _{n}\left\langle f, a_{n} b_{m}\right\rangle
$$

whenever both iterated limits exist. The collection of the wap functionals on $A$ is denoted by $\operatorname{wap}(A)$. The $\operatorname{set} \operatorname{wap}(A)$ is a closed subspace of $A^{*}$ and the equality $\operatorname{wap}(A)=A^{*}$ is equivalent to the Arens regularity of $A$ [41].

Our basic references about the RNP are the books [6] and [12]. We refer the reader to these books for relevant definitions. The following well-known results will be sufficient for our purposes. The dual $X^{*}$ of a Banach space $X$ has the RNP iff any separable subspace $Y$ of $X$ has separable dual, see [6, Cor. 4.1.7] and [12, Cor. VII.2.8]. Here note that if $A$ is a Banach algebra then $A^{*}$ has the RNP iff any separable subalgebra $B$ of $A$ has separable dual. Indeed, the necessity of this condition is obvious and the sufficiency follows from the facts that if $Y$ is a separable subspace of $A$ then the subalgebra $B$ of $A$ generated by $Y$ is separable and the separability of $B^{*}$ implies that of $Y^{*}$. We shall use this characterization several times. A weak*-compact convex subset $M$ of the dual space $X^{*}$ of a Banach space $X$ has the RNP iff, for each separable subspace $Y$ of $X$, the set of restrictions $\left.M\right|_{Y}=\left\{\left.f\right|_{Y}: f \in M\right\}$ is a separable subset of $Y^{*}$ [6, Thm. 4.2.13]. In particular, any separable weak ${ }^{*}$-compact convex and any convex

\footnotetext{
${ }^{1}$ Although our definition of BLAI includes condition $\left\|e_{\alpha}\right\| \leq 1$ all the results presented in this note hold for an arbitrary BLAI.
} 
weakly compact subsets of $X^{*}$ have the RNP, see [6, Prop. 4.1.1] and [6, Thm. 3.6.1], respectively.

Throughout the paper by a linear (bilinear) operator we mean a bounded linear (bilinear) operator.

3. The main results. This section contains the main results of this note. These results apply to Banach algebras having a bounded left (or right) approximate identity. We shall work with a Banach algebra having a BLAI. Let $A$ be a Banach algebra with a BLAI $\left(e_{\alpha}\right)_{\alpha \in I}$. We introduce the following subspace of $A$.

$$
l\left(A^{*}\right)=\left\{f: f \in A^{*} \text { and } e_{\alpha} f \rightarrow f \text { weakly }\right\} .
$$

The following theorem shows that the space $l\left(A^{*}\right)$ does not depend on the BLAI chosen and that, in the case where $A$ is a right ideal in its second dual, $l\left(A^{*}\right)=\operatorname{wap}(A)$.

TheOREM 3.1. Let $A$ be a Banach algebra with a BLAI $\left(e_{\alpha}\right)_{\alpha \in I}$. Then,

(a) $l\left(A^{*}\right)=A A^{*}$ and $\operatorname{wap}(A)$ is contained in $l\left(A^{*}\right)$.

(b) If $A$ is a right ideal in its second dual, $\operatorname{wap}(A)=l\left(A^{*}\right)$.

Proof. (a) We first note that, by the Cohen-Hewitt factorization theorem $[32,32.22]$, the set $A A^{*}$ is a closed subspace of $A^{*}$. Since each $e_{\alpha} f$ is in $A A^{*}$, the inclusion $l\left(A^{*}\right) \subseteq A A^{*}$ is obvious. To prove the reverse inclusion, let $a$ be in $A$ and $f$ be in $A^{*}$. Then

$$
\left\|e_{\alpha} a f-{ }_{a} f\right\|=\left\|_{\left(e_{\alpha} a-a\right)} f\right\| \leq\|f\|\left\|e_{\alpha} a-a\right\| \rightarrow 0 .
$$

In particular, $e_{\alpha} f f \rightarrow{ }_{a} f$ weakly, and $l\left(A^{*}\right)=A A^{*}$.

To show that the space $\operatorname{wap}\left(A^{*}\right)$ is contained in $l\left(A^{*}\right)$, let $f$ be a wap functional on $A$. Then the set $\left\{e_{\alpha} f: \alpha \in I\right\}$ is relatively weakly compact and, since $\left(e_{\alpha}\right)$ is a BLAI, $e_{\alpha} f \rightarrow f$ for the weak* topology. This implies that $f$ is the unique weak cluster point of the net $\left(e_{\alpha} f\right)$, which is contained in a relatively weak compact set. It follows that $e_{\alpha} f \rightarrow f$ weakly, and $\operatorname{wap}(A) \subseteq l\left(A^{*}\right)$.

(b) Assume $A$ is a right ideal in its second dual. Then, for each $a$ in $A$, the left multiplication operator $a^{\tau}$ is weakly compact [13; p. 318, Lemma 3]. Let $a$ be in $A$ and $f$ be in $A^{*}$. For each $y$ in $A$, let $f_{y}$ be the functional on $A$ defined by $f_{y}(x)=f(x y)$. The set $K=\left\{{ }_{a} f_{y}: y \in A_{1}\right\}$, being the image under ${ }_{a} \tau^{*}$ of the set $\left\{f_{y}: y \in\right.$ $\left.A_{1}\right\}$, is relatively weakly compact. It follows that the functional af is wap on $A$, and the equality $\operatorname{wap}(A)=A A^{*}$ holds.

The following corollary is immediate from this theorem. For another proof of it, see [24; Lemma 4 and Cor.]. 
Corollary 3.2. Let $A$ be a Banach algebra with a BAI. Assume $A$ is a bisided ideal in its second dual. Then $A A^{*}=A^{*} A$ and, if $A$ is regular, $A^{*}=A A^{*}=A^{*} A$.

As an illustration of Theorem 3.1 we give the following proposition which we shall need in the next section.

Proposition 3.3. Let $G$ be a locally compact topological group equipped with a left Haar measure and $A=L^{1}(G)$ be the group algebra of $G$. Then $l\left(A^{*}\right)=A A^{*}=\operatorname{LUC}(G)$ and $A^{*} A=\operatorname{RUC}(G)$.

Proof. We shall prove the equality $A A^{*}=\operatorname{LUC}(G)$, the proof of the equality $A^{*} A=\operatorname{RUC}(G)$ is very similar. For $u$ in $L^{1}(G)$, let $\tilde{u}(t)=\Delta\left(t^{-1}\right) u\left(t^{-1}\right)$, where $\Delta$ is the modular function of the group $G$. An easy calculation will show that (see [59]), for $f$ in $A^{*}, u f=$ $\tilde{u} * f$ where $*$ denotes the convolution. Since the operator $u \rightarrow \tilde{u}$ is an involutive isometry from $L^{1}(G)$ onto itself, we have the equality $A A^{*}=L^{1}(G) * L^{\infty}(G)$. Since by $[32 ; 32.45(\mathrm{~b})], L^{1}(G) * L^{\infty}(G)=$ LUC $(G)$, we conclude that $A A^{*}=\operatorname{LUC}(G)$.

The next lemma will permit us to assume that our algebra is separable and its BLAI is a sequence. In this lemma the subalgebras considered need not be closed.

Lemma 3.4. Let $A$ be a Banach algebra with a BLAI $\left(e_{\alpha}\right)_{\alpha \in I}$. Then, given any separable subalgebra $B_{0}$ of $A$, there exists a separable subalgebra $B$ containing $B_{0}$ and having a sequential $B L A I\left(w_{n}\right)_{n \in N}$. Moreover, if each set $H_{\alpha}=\left\{e_{\alpha} f: f \in A_{1}^{*}\right\}$ has the RNP then each set $\widetilde{H}_{n}=\left\{w_{n} g: g \in B_{1}^{*}\right\}$ is separable.

Proof. Let $B_{0}$ be a separable subalgebra of $A$ and $\left(b_{n}\right)$ a dense sequence in $B_{0}$. For each $n=0,1,2, \ldots$, choose an $\alpha_{n}$ in $I$ such that we have:

$$
\left\|e_{\alpha_{n}} b_{i}-b_{i}\right\|<\frac{1}{n+1} \quad \text { for } 0 \leq i \leq n .
$$

Let $B_{1}$ be the subalgebra generated by the union of $B_{0}$ and the set $\left\{e_{\alpha_{n}}: n \in N\right\}$. Then $B_{1}$ is a separable subalgebra of $A$ and, repeating for $B_{1}$ what we have done for $B_{0}$, we obtain a separable subalgebra $B_{2}$ containing $B_{1}$. Continuing this process we get an increasing sequence $\left(B_{n}\right)$ of separable subalgebras. Let $B$ be the union of them. 
Then $B$ is a separable subalgebra of $A$ and it contains $B_{0}$. Let $U$ denote the set of all the $e_{\alpha_{n}}$ 's involved in the construction of $B$. The set $U$ is countable and for any $\varepsilon>0$ and any $b$ in $B$ there is an $e_{\alpha}$ in $U$ such that $\left\|e_{\alpha} b-b\right\|<\varepsilon$. Now let $W=\left\{e_{\alpha} e_{\beta}: e_{1}, e_{\beta}\right.$ are in $\left.U\right\}$. The set $W$ is countable and the proof of Proposition 2 of $[4 ;$ p. 58] shows that from $W$ we can extract a sequence, say $\left(w_{n}\right)$, which is a BLAI for $B$. To prove the last assertion of the lemma, assume that each set $H_{\alpha}$ has the RNP. Let $e_{\beta} \tau^{*}$ be the adjoint of the left multiplication operator $e_{\beta} \tau: A \rightarrow A$. Since $e_{\beta} \tau^{*}\left(H_{\alpha}\right)=\left\{e_{\beta}\left(e_{\alpha} f\right): f \in A_{1}^{*}\right\}$, by Corollary 4.2.14 of [6], the set $\left\{\left(e_{\alpha} e_{\beta}\right) f: f \in A_{1}^{*}\right\}$ has the RNP. It follows that for any $w$ in $W$ the set $\left\{w f: f \in A_{1}^{*}\right\}$ has the RNP. Hence, for each $n$, the set $\left\{w_{n} g: g \in B_{1}^{*}\right\}$, which is just the set $\left.\left\{w_{n} f: f \in A_{1}^{*}\right\}\right|_{B}$, is separable.

Now, we can prove the main result of this note.

Theorem 3.5. Let $A$ be a Banach algebra with a BLAI $\left(e_{\alpha}\right)_{\alpha \in I}$. Assume that, for each $\alpha \in I$, the set $H_{\alpha}=\left\{e_{\alpha} f: f \in A_{1}^{*}\right\}$ has the RNP. Then, for each separable subalgebra $B_{0}$ of $A$, the space $\left.\operatorname{wap}(A)\right|_{B_{0}}$ is separable. In particular, if $A$ is regular, $A^{*}$ has the $R N P$.

Proof. Let $B_{0}$ be a separable subalgebra of $A$. By the preceding lemma there exists a separable algebra $B$ containing $B_{0}$ and having a sequential BLAI $\left(w_{n}\right)$ such that each set $\widetilde{H}_{n}=\left\{w_{n} g: g \in B_{1}^{*}\right\}$ is separable. It is clear that the separability of the $\left.\operatorname{space} \operatorname{wap}(A)\right|_{B}$ implies that of the $\left.\operatorname{space} \operatorname{wap}(A)\right|_{B_{0}}$ and that we have the inclusion $\left.\operatorname{wap}(A)\right|_{B} \subseteq \operatorname{wap}(B)$. Therefore it is enough to prove that the space $\operatorname{wap}(B)$ is a separable subspace of $B^{*}$. To this end for each $n \in \mathbb{N}$, let $E_{n}=\left\{w_{n} g: g \in B_{1}^{*}\right.$ and $g$ is wap on $\left.B\right\}$. Since $E_{n} \subseteq \widetilde{H}_{n}$ and $\widetilde{H}_{n}$ is separable, the set $E_{n}$ is separable. Let $E$ be the convex hull of the union of $E_{n}$ 's. We are going to show that $E$ is weakly (so norm) dense in the closed unit ball of the space wap $(b)$. To this end let $g$ be wap on $B$ with $\|g\| \leq 1$ and $b^{* *}$ be an arbitrary element of $B^{* *}$. By the Goldstine Theorem, there exists a bounded net $\left(b_{\beta}\right)$ in $B$ which converges weak ${ }^{*}$ to $b^{* *}$. Now, since $g$ is wap on $B$ an $\left(w_{n}\right)$ is a BLAI for $B$, we have

$$
\begin{aligned}
\left\langle g, b^{* *}\right\rangle & =\lim _{\beta}\left\langle g, b_{\beta}\right\rangle=\lim _{\beta} \lim _{n}\left\langle g, w_{n} b_{\beta}\right\rangle \\
& =\lim _{n} \lim _{\beta}\left\langle g, w_{n} b_{\beta}\right\rangle=\lim _{n} \lim _{\beta}\left\langle_{w_{n}} g, b_{\beta}\right\rangle \\
& =\lim _{n}\left\langle w_{n} g, b^{* *}\right\rangle .
\end{aligned}
$$


This shows that the sequence $\left(w_{n} g\right)$, which is contained in $E$, converges weakly to $g$. Hence the set $E$, which is separable, is dense in the closed ball of the $\operatorname{space} \operatorname{wap}(B)$. To prove the last assertion of the theorem, assume that $A$ is regular. Then $\operatorname{wap}(A)=A^{*}$ and for any separable subalgebra $B$ of $A, B^{*}=\left.\operatorname{wap}(A)\right|_{B}$ is separable. It follows that $A^{*}$ has the RNP.

This theorem has several corollaries. The first corollary is a nonembedding result. In the next section we shall see a concrete application of it.

Corollary $3.6^{1}$. Let $A$ be a Banach algebra with a BLAI $\left(e_{\alpha}\right)_{\alpha \in I}$. Assume that, for each $\alpha$ in $I$, the set $H_{\alpha}$ has the RNP. Then, if $X$ is a separable subspace of $A$ with $X^{*}$ unseparable, the space wap $(A)$ does not contain a (isomorphic) copy of $X^{*}$.

Proof. Let $X$ be a separable subspace of $A$ and $B$ the subalgebra generated by $X$. The subalgebra $B$ is separable and the natural restriction operator from $\left.\operatorname{wap}(A)\right|_{B}$ to $\left.\operatorname{wap}(A)\right|_{X}$ is onto, so that the space $\left.\operatorname{wap}(A)\right|_{B}$ has a quotient isomorphic to $\left.\operatorname{wap}(A)\right|_{X}$. Should the space $\operatorname{wap}(A)$ contain a copy of $X^{*}$, the space $\left.\operatorname{wap}(A)\right|_{X}$ would contain a copy of $X^{*}$ and be unseparable. But then $\left.\operatorname{wap}(A)\right|_{B}$ cannot be separable. From this we conclude that the space wap $(A)$ does not contain a copy of $X^{*}$.

The only hypothesis in the statement of Theorem 3.5 which may be difficult to verify in practice is the condition that, for each $\alpha$ in $I$, the set $H_{\alpha}$ should have the RNP. However there exists an important class of Banach algebras for which this condition is automatically satisfied. Indeed, if $A$ is a right ideal in its second dual then for each $a$ in $A$, the set $H(a)=\left\{a f: f \in A_{1}^{*}\right\}$ is weakly compact $[13 ;$ p. 315 , Lemma 3], so has the RNP [6; Thm. 3.6.1]. The next corollary is now immediate.

Corollary 3.7. Let $A$ be a Banach algebra with a BLAI. If $A$ is regular and a right ideal in its second dual then $A^{*}$ has the RNP.

As an application of this corollary we give the following result. In the next section we shall see some other applications of it.

\footnotetext{
${ }^{1}$ See note added in proof.
} 
COROllary 3.8. Let $A$ be a $B^{*}$-algebra which is also a dual algebra. Then $A^{*}$ has the RNP.

Proof. Any $B^{*}$-algebra has a BLAI $[4 ;$ p. 218, Lemma 14] and is regular [10; Thm. 7.1]. $A$, being a dual algebra, is a bisided ideal in its second dual [50; p. 533, Thm. 5.1]. Hence, by the preceding corollary, $A^{*}$ has the RNP.

The next corollary applies to WSC (weakly sequential complete) Banach algebras. We note that several Banach algebras of Harmonic Analysis are WSC.

COROllary 3.9. Let $A$ be a WSC Banach algebra with a BLAI $\left(e_{\alpha}\right)_{\alpha \in I}$. Assume that, for each $\alpha$ in $I$, the set $H_{\alpha}$ has the RNP. Then $A$ is regular iff $A$ is reflexive.

Proof. Assume $A$ is regular. Then, by Theorem 3.5, $A^{*}$ has the RNP. Therefore $A$ does not contain a copy of $l^{1}$ since $l^{\infty}$ is not separable. But, by [45; p. 807, Cor. 1], a WSC Banach space not containing a copy of $l^{1}$ is reflexive. Hence $A$ is reflexive. The converse is trivial.

Before stating the next theorem we recall that a Banach space $X$ is said to have the DPP (Dunford-Pettis Property) if any weakly compact operator from $X$ into a Banach space $Y$ sends weakly convergent sequences into norm convergent ones. It is well known that any $L^{1}(\mu)$ space, any $C(K)$ space and the disk algebra $A(D)$ have the DPP, see [11] and [27]. We also recall that a Banach space $X$ is said to have the Schur property if in $X$ any weakly convergent sequence is norm convergent. The next theorem characterizes the Arens regularity of a rather restrictive class of Banach algebras.

Theorem 3.10. Let $A$ be a Banach algebra with a BLAI $\left(e_{\alpha}\right)_{\alpha \in I}$. Assume that, for each $\alpha \in I$, the set $H_{\alpha}$ has the RNP. Then $A$ is regular and has the DPP iff $A^{*}$ has the RNP and the Schur property.

Proof. Assume $A$ is regular and has the DPP. Then, by Theorem $3.5 A^{*}$ has the RNP. It follows that $A$ does not contain a copy of $l^{1}$. Hence, since $A$ has the DPP, by [11; p. 23, Thm. 3] $A^{*}$ has the Schur property.

Conversely, assume that $A^{*}$ has the RNP and the Schur property. Then $A^{*}$ also has the DPP and therefore, so has $A[11 ;$ p. 29, Cor. 2]. 
To prove that $A$ is regular it is enough to apply the following lemma, which is of independent interest.

LeMma 3.11. Let $X$ and $Y$ be two Banach spaces such that $X^{*}$ has the RNP and $Y^{*}$ is WSC. Then, for any Banach space $Z$, any bilinear operator $m: X \times Y \rightarrow Z$ is Arens regular.

Proof. By [2; Thm. 3.2] it is enough to prove that any bilinear form $m: X \times Y \rightarrow \mathbb{C}$ is Arens regular. But by [53] this is equivalent to proving that every linear operator $u: X \rightarrow Y^{*}$ is weakly compact. Now, let $u: X \rightarrow Y^{*}$ be a linear operator and $\left(x_{n}\right)$ a bounded sequence in $X$. Since $X^{*}$ has the RNP, $X$ does not contain a copy of $l^{1}$ and by Rosenthal's $l^{1}$-theorem [45; Thm. 3] $\left(x_{n}\right)$ has a weakly Cauchy subsequence. The space $Y^{*}$ being WSC, we conclude that the sequence $\left(u\left(x_{n}\right)\right)$ has a weakly convergent subsequence, and $u$ is weakly compact.

Now, let $G$ be a locally compact group equipped with a left Haar measure and $A=L^{1}(G)$ be the group algebra of $G$. The algebra $A$ has a BAI and has the DPP. So has the algebra $A=C_{0}(T)$, where $T$ is any locally compact space. Akermann has shown that $[1$; Thm. 4] if $G$ is compact then, for any $a$ in $L^{1}(G)$, the left multiplication operator ${ }_{a} \tau$ is compact. In [18] $\mathrm{F}$. Ghahramani has extended this result to weighted group algebras constructed over compact topological groups. The next result, which we shall need in the next section, shows that the above-mentioned results are particular cases of the following proposition.

Proposition 3.12. Let $A$ be a Banach Algebra with a BLAI and the DPP. Then the following two assertions are equivalent.

(i) $A$ is a right ideal in its second dual.

(ii) For any $a$ in $A$, the operator a $\tau$ is compact.

Proof. In view of $[13 ;$ p. 318 , Lemma 3$]$ it is enough to show that (i) $\rightarrow$ (ii). Let $a \in A$. By Cohen's factorization theorem [32; 32.26] there exist $b$ and $c$ in $A$ such that $a=b c$. Hence ${ }_{a} \tau={ }_{b} \tau{ }^{\circ} \tau$. Since by hypothesis, the operators ${ }_{b} \tau$ and ${ }_{c} \tau$ are weakly compact and $A$ has the DPP, $a^{\tau}$ is compact.

The next result completes in a way the preceding proposition and shows that if the carrier space $[4 ;$ p. 81] of a commutative Banach algebra $A$ is connected then $A$ has little chance to be an ideal in its second dual. 
Proposition 3.13. Let $A$ be a commutative semisimple complex Banach algebra whose carrier space $\Phi$ is connected. Then the only element $a$ in $A$ for which the operator a $\tau$ is compact is $a=0$. If, moreover, $A$ has the DPP then $a=0$ is the only element in $A$ for which the operator ${ }_{a} \tau$ is weakly compact.

Proof. Let $a$ be an element in $A$ for which ${ }_{a} \tau$ is compact. Denote by $\hat{a}$ the functional on $A^{*}$ defined by $\hat{a}(f)=f(a)$. Since the space $\Phi$ is connected and $\hat{a}$ is weak ${ }^{*}$-continuous, $\hat{a}(\Phi)=\{f(a): f \in \Phi\}$ is a connected subset of $\mathbb{C}$. Now, as it is immediate to see, for any $f$ in $\Phi,{ }_{a} \tau^{*}(f)=f(a) f$ so that $f(a)$ is an eigenvalue of the operator ${ }_{a} \tau^{*}$, which is compact. Since the spectrum of a compact linear operator is at most countable [14; VII.4.5] and $\hat{a}(\Phi)$ is contained in the spectrum of the operator $a_{a} \tau^{*}$ we conclude that $\hat{a}(\Phi)=\{\lambda\}$ for some $\lambda$ in $\mathbb{C}$. We claim that $\lambda=0$. Indeed $\lambda \neq 0$ implies, by [4; p. 84, Prop. 9], that $A$ is unital and $a$ is invertible. But this is not possible since ${ }_{a} \tau$ is compact. Hence $\lambda=0$ and the semisimplicity of $A$ implies that $a=0$. Now assume $A$ has the DPP and for some $a$ in $A,{ }_{a} \tau$ is weakly compact. Then $a^{2} \tau={ }_{a} \tau \circ_{a} \tau$ is compact and so $a^{2}=0$ by what precedes. Again by semisimplicity of $A$ we conclude that $a=0$.

We note that the preceding proposition applies, e.g., to the algebras $C([0,1]), C_{0}(\mathbb{R}), L^{1}(\mathbb{R}), l^{1}(\mathbb{Z})$ and that these algebras have the DPP.

We close this section with some remarks and examples.

REMARKS AND EXAMPLES 3.14. (a) Let $J$ be the James space [37; p. 25] and $A=K(J)$ the algebra of compact operators on $J$. Since $J$ has a Schauder basis and $J^{* *}$ is isometrically isomorphic to $J$, the spaces $J, J^{*}$ and $J^{* *}$ have the a.p. and they are separable. Hence $A^{*}=J^{*} \hat{\otimes} J^{* *}$ and $A^{* *}=L\left(J^{* *}\right)\left[12 ;\right.$ p. 248 , Thm. 6]. Since $A^{*}$ is separable it has the RNP. Moreover $A$ has a BLAI since $A^{* *}$ has a unit element [10; Lemma 3.8]. But $A$ is not regular since $J$ is not reflexive ([38], [54] or [62]). This example shows that the converse of Theorem 3.5 is not true.

(b) Let $T$ be a locally compact space and $A=C_{0}(T)$. S. L. Gulick has shown that [29; Thm. 4.9] $A$ is an ideal in its second dual iff $T$ is discrete. (A short proof of this result goes as follows: Assume $A$ is an ideal in $A^{* *}$. Then so is any quotient algebra of $A$. Now, let $F$ be any compact subset of $T$. Since the algebra $C(F)$ is isomorphic to a quotient of $A$ and it has the DPP and is unital, by Proposition 3.12 the identity operator on $C(F)$ is compact. But this is possible only if $F$ is finite. It follows that $T$ is discrete. The converse is 
immediate.) Now, assume that $T$ is noncompact dispersed (i.e. has no nonempty perfect subset) and nondiscrete. Such spaces exist, for example an infinite appropriate ordinal space, see [48; pp. 149-151]. Then the algebra $C_{0}(T)$ is regular, has a BAI and the DPP, its dual space has the RNP [39] had the Schur property [11, Thm. 3]. But $C_{0}(T)$ is not an ideal in its second dual. On the other hand, as we shall see below, the group algebra $L^{1}(G)$ of an infinite compact group is an ideal in its second dual but it is neither regular nor has its dual the RNP. These examples show that the property that an algebra $A$ is an ideal in its second dual is independent of the geometric properties of Banach spaces involved so far in this note.

4. Applications I. In this section we present some applications of the results given in $\S 3$ to topological groups, group algebras and their wap functionals.

Let $G$ be a locally compact topological group and $A=L^{1}(G)$ be its group algebra with respect to a left Haar measure of $G$. The algebra $A$ has a BAI [31; p. 303] and it is unital if $G$ is discrete. We give without proof the following well-known result which is an easy application of the Dunford-Pettis criterion [14; p. 294, Cor. 11] characterizing the weakly compact subsets of $L^{1}(G)$.

Lemma 4.1. Assume $G$ is compact. Then the algebra $A=L^{1}(G)$ is a bisided ideal in its second dual.

The following result, in the case where $G$ is locally compact and commutative, is due to Civin and Yood [10]. N. Young has extended it to noncommutative groups [61]. Below we shall present four short proofs of this proposition. Some other proofs, partial or complete, of this result can be found in the papers [51], [55] and [60].

Proposition 4.2. Assume $G$ is compact. Then the algebra $A=$ $L^{1}(G)$ is regular iff $G$ is finite.

Proofs. 1. This proof is based on Theorem 3.1.(b) above. By this theorem and Proposition 3.3, $\operatorname{wap}(A)=A A^{*}=C(G)$. Therefore $A$ is regular iff $L^{\infty}(G)=C(G)$ iff $G$ is finite.

2. This proof is based on Corollary 3.9. By this corollary $A$ is regular iff it is reflexive. This is possible iff $G$ is finite.

3. This proof is based on Theorem 3.10. By this theorem $A$ is regular iff $A^{*}=L^{\infty}(G)$ has the RNP and the Schur property. This is possible iff $G$ is finite. 
4. This proof is a little bit longer than the preceding ones but it does not use Lemma 4.1 above. We shall apply Theorem 3.5 directly. First note that $G$ has a metrizable quotient group $G / H[31 ; 8.7]$, where $H$ is a closed normal subgroup of $G$. On the other hand, the group algebra of $G / H$ is isometrically isomorphic to a quotient algebra of the algebra $L^{1}(G)[44 ;$ p. $74,5.3]$ and quotient algebras of regular algebras are regular. Therefore we can assume that $G$ is metrizable so that the spaces $L^{1}(G)$ and $C(G)$ are both separable. Now, let $\left(e_{n}\right)_{n \in \mathbb{N}}$ be a BAI of $A$. For each $n \in \mathbb{N}$, the set $H_{n}=\left\{e_{n} f: f \in\right.$ $\left.A_{1}^{*}\right\}$ is contained in $C(G)$ (see the proof of Proposition 3.3 above). Hence each $H_{n}$ is separable, so has the RNP. Should $A$ be regular, by Theorem 3.5, $A^{*}=L^{\infty}(G)$ would have the RNP. But this is possible only if $G$ is finite. The converse is trivial.

REMARK 4.3. Assume $G$ is locally compact and nondiscrete, and $A=L^{1}(G)$. Theorem 3.1(a) shows that $\operatorname{wap}(A) \subseteq A A^{*}=\operatorname{LUC}(G)$. Therefore, should $L^{1}(G)$ be regular we would have $L^{\infty}(G)=\operatorname{LUC}(G)$, which is not possible. This remark shows that, concerning the regularity of $L^{1}(G)$, the only case which is not covered by Theorem 3.1 is the one where $G$ is discrete.

The next result is about the location of the space $\operatorname{wap}\left(L^{1}(G)\right)$ inside $L^{\infty}(G)$. In [15] and [55] it is shown that, for any locally compact group, wap $\left(L^{1}(G)\right)=\operatorname{WAP}(G)$. On the other hand, Burckel has shown that the equality $\operatorname{WAP}(G)=C(G)$ holds iff $G$ is compact [7; p. 68, Thm. 4.10]. Later, this result has been improved by several authors, see e.g. [8], [16] and [22]. The best result in this direction seems to be the following [16; Cor. 2.2]: if $G$ is not compact then the quotient space $\operatorname{UC}(G) / \mathrm{WAP}(G)$ contains an isometric copy of $l^{\infty}$. Below, as an application of Theorem 3.1, we give a short proof of some of these results.

Proposition 4.4. Let $G$ be locally compact. Then $\operatorname{wap}\left(L^{1}(G)\right) \subseteq$ $\mathrm{UC}(G)$. Moreover, if $G$ is not discrete, the equality $\operatorname{wap}\left(L^{1}(G)\right)=$ $C(G)$ holds iff $G$ is compact.

Proof. Since the algebra $A=L^{1}(G)$ has a bisided approximate identity, Theorem 3.1(a) and Proposition 3.3 show that the space $\operatorname{wap}(A)$ is contained in both spaces $\operatorname{LUC}(G)$ and $\operatorname{RUC}(G)$. Hence $\operatorname{wap}(A) \subseteq$ $\mathrm{UC}(G)$. If $G$ is compact, then, by Theorem 3.1(b), wap $(A)=C(G)$. Conversely, assume that we have $\operatorname{wap}(A)=\operatorname{UC}(G)$. Then $C(G)=$ wap $(A)=\mathrm{UC}(G)$. But for a locally compact nondiscrete topological 
group, the equality $C(G)=\mathrm{UC}(G)$ is possible only if $G$ is compact [36; Cor. 2].

The next result is an application of Corollary 3.6. We do not know whether this result is known or not.

Proposition 4.5. Let $G$ be a compact topological group. Then the space $C(G)$ does not contain a (isomorphic) copy of $l^{\infty}$.

Proof. Assume $G$ is infinite and let $A=L^{1}(G)$ be its group algebra. Then the algebra $A$ is irregular. Let $B$ be any nonreflexive separable closed subalgebra of $A$. (Such a $B$ exists since $A$ is not regular; recall that a Banach algebra is regular iff every separable subalgebra of it is regular.) The space $B$, being WSC, contains a copy of $l^{1}$ [45; Cor. 1]. Since $l^{\infty}=\left(l^{1}\right)^{*}$ is unseparable, by Proposition 4.4 and Corollary 3.6 we conclude that $C(G)=\operatorname{wap}(A)$ does not contain a copy of $l^{\infty}$.

As a corollary of this proposition we give a short proof of the following result due to Rajagopalan [43]. For other proofs of it, see [23; p. 322, Prop.] and [29; Lemma 3.9].

Corollary 4.6. Let $G$ be a compact topological group. Then $G$ is extremally disconnected iff $G$ is finite.

Proof. Assume $G$ is infinite and extremally disconnected. Then, by a result of James [34], $C(G)$ contains an isomorphic copy of $l^{\infty}$. This being impossible by the preceding proposition we conclude that every extremally compact topological group is finite. The converse is trivial.

REMARK 4.7. Let $K$ be an infinite compact space. It is known that if $K$ is $\sigma$-Stonean (i.e. disjoint open subsets of $K$, at least one of which is an $F_{\sigma}$, have disjoint closures) or if $K$ is an $F$-space (i.e. disjoint open $F_{\sigma}$ subsets of $K$ have disjoint closures) see [47], then the space $C(K)$ contains an isomorphic copy of $l^{\infty}$ (in the case where $K$ is an $F$-space the Continuum Hypothesis is assumed), see the introduction of the papers [30] and [49]. From this and the proof of the preceding corollary we conclude that a compact topological group is $\sigma$-Stonean or an $F$-space iff it is finite.

The next result is due to Watanabe ([57], [58]). Some other proofs, partial or complete, of this result can be found in [9], [19], [25], [33] and [35]. 
Proposition 4.8. Let $G$ be a locally compact topological group and $A=L^{1}(G)$ be its group algebra. Then $a$ is a right ideal in its second dual iff $G$ is compact.

Proof. Assume $A$ is a right ideal in its second dual. Then the main hypothesis of Corollary 3.6 is satisfied so that the $\operatorname{space} \operatorname{wap}(A)$-does not contain a copy of $l^{\infty}$. On the other hand, by Theorem 3.1(a), $\operatorname{wap}(A)=\operatorname{LUC}(G)$ and it is well known that, if $G$ is not compact, the space $\operatorname{LUC}(G)$ contains an isometric copy of $l^{\infty}$, see, e.g., the proof of Theorem 2.1 of [16]. From this contradiction we deduce that $G$ is compact. The converse is the object of Lemma 4.1 above.

The next remark contains two other proofs of this proposition. Yet another proof can be obtained from Proposition 3.13 above if the group $G$ has an abelian quotient group whose dual group is connected.

Remarks 4.9. (a) S. Sakai [46; Thm. 1] has shown that if $G$ is not compact then the only element $a$ in $L^{1}(G)$ for which the left multiplication operator $a \tau$ is compact is $a=0$. This result combined with Proposition 3.12 above furnishes another proof of the preceding result.

(b) Assume $G$ has an infinite discrete quotient group $G / H$. This is the case for example if $G$ is noncompact, compactly generated and Abelian $[31 ; 9.8]$. Then the group algebra of $G / H$ is a unital infinite dimensional Banach algebra with the DPP. Therefore, by Proposition 3.12, $L^{1}(G / H)$ cannot be a right ideal in its second dual nor can $L^{1}(G)$ be since $L^{1}(G / H)$ is isometrically isomorphic to a quotient algebra of it. This argument furnishes, in this particular case, another proof of Proposition 4.8.

5. Applications II. In this section we give some applications of the results given in $\S 3$ to the injective and projective tensor products of two Banach spaces and algebras.

If $X$ and $Y$ are two Banach spaces having the RNP their projective tensor product $X \hat{\otimes} Y$ need not have the RNP. Indeed, in [5] J. Bourgain and $\mathrm{G}$. Pisier have given an example of Banach space $X$ which has the RNP and is WSC but the space $X \hat{\otimes} X$ contains a copy of $c_{0}$. On the positive side, if both $X^{*}$ and $Y^{*}$ have the RNP and one of them has the a.p. then the space $X^{*} \hat{\otimes} Y^{*}$ has the RNP $[12 ;$ p. 249 , Thm. 7]. The next proposition is a particular case of this result and is obtained as an application of Corollary 3.7 above.

Proposition 5.1. Let $X$ and $Y$ be two reflexive Banach spaces with the a.p. Then the space $X \otimes Y$ has the RNP. 
Proof. Put $Z=X \times Y^{*}$. Equipped with the norm $\left\|\left(x, y^{*}\right)\right\|=$ $\|x\|+\left\|y^{*}\right\|, Z$ is a reflexive Banach space and has the a.p. Consider the operator algebra $K(Z)=Z^{*} \hat{\otimes} Z$ of the compact linear operators on $Z$. Since $Z$ is reflexive and has the a.p., $K(Z)^{*}=Z \hat{\otimes} Z^{*}$ and $K(Z)^{* *}=L(Z)$, see [12; p. 248, Thm. 6 and Thm. 7]. The algebra $K(Z)$ is regular ([38], [54] and [62]) and is a bisided ideal in its second dual. Since the algebra $L(Z)$ is unital, $K(Z)$ has a BAI [10; Lemma 3.8] (see also [38; Thm. 2]). Therefore by Theorem 3.5 (or Corollary 3.7) $K(Z)^{*}=Z \hat{\otimes} Z^{*}$ has the RNP. Now, it is clear that $X$ (resp. $Y$ ) is complemented in $Z$ (resp. $Z^{*}$ ) by a norm-one projection. Therefore we can regard $X \hat{\otimes} Y$ as a subspace of $Z \hat{\otimes} Z^{*}$, see [28; p. 39, Prop. $4(2)]$. It follows that the space $X \hat{\otimes} Y$ also has the RNP.

The next result is about the Arens regularity of the projective tensor product algebra $A \hat{\otimes} B$ of two Banach algebras $A$ and $B$ with BLAI. We recall that on $A \hat{\otimes} B$ the multiplication is the linear extension of the following natural multiplication on decomposable tensors

$$
(a \otimes b)(\tilde{a} \otimes \tilde{b})=a \tilde{a} \otimes b \tilde{b} .
$$

For more information about the tensor product of Banach algebras the reader may consult Chapter VI of the book [4] and the papers [17], [20] and [52], and about the regularity of the algebra $A \hat{\otimes} B$, the paper [56]. Now let $A$ and $B$ be two Banach algebras with BLAI $\left(e_{\alpha}\right)_{\alpha \in I}$ and $\left(d_{\beta}\right)_{\beta \in J}$, respectively. It is easy to see that the net $\left(e_{\alpha} \otimes d_{\beta}\right)_{(\alpha, \beta) \in I \times J}$ is a BLAI for the algebra $A \hat{\otimes} B$. On the other hand, even if both $A$ and $B$ are reflexive and commutative the algebra $A \hat{\otimes} B$ need not be an ideal in its second dual. here is a simple example.

EXAMPLE 5.2. Let $A=l^{2} \oplus \mathbb{C}$ be the unitization $[4 ; \mathrm{p} .15]$ of the usual Hilbert space $l^{2}$ endowed with the coordinatewise multiplication. Then the algebra $A \hat{\otimes} A$ is unital and nonreflexive; therefore it cannot be an ideal in its second dual.

We recall that the dual of $A \hat{\otimes} B$ can be identified with the space $L\left(A, B^{*}\right)$ [12; Chapter VIII] so that, for $S$ in $L\left(A, B^{*}\right), a, x$ in $A$ and $b, y$ in $B$, we have:

$$
\begin{array}{r}
\langle a \otimes b \\
\quad, x \otimes y\rangle=\langle S, a \otimes b . x \otimes y\rangle=\langle S, a x \otimes b y\rangle \\
\quad=\left\langle S,{ }_{a} \tau(x) \otimes{ }_{b} \tau(y)\right\rangle=\left\langle{ }_{b} \tau^{*} \circ S \circ_{a} \tau, x \otimes y\right\rangle .
\end{array}
$$

Thus ${ }_{a \otimes b} S={ }_{b} \tau^{*} \circ S \circ{ }_{a} \tau$. Now let $u=\sum_{n=1}^{\infty} a_{n} \otimes b_{n}$ be a general 
element of $A \hat{\otimes} B$. For such a $u$, we have

$$
u S=\sum_{n=1}^{\infty} b_{n} \tau^{*} \circ S \circ{ }_{a_{n}} \tau .
$$

Finally, recall that $A \hat{\otimes} B \quad L\left(A, B^{*}\right)$ denotes the space of $u S$ 's for $u$ in $A \hat{\otimes} B$ and $S$ in $L\left(A, B^{*}\right)$.

Except the first inclusion of the first assertion, the following theorem seems to be new.

Theorem 5.3. Let $A$ and $B$ be two regular Banach algebras with $B L A I$. Then we have:

(i) $K\left(A, B^{*}\right) \subseteq \operatorname{wap}(A \hat{\otimes} B) \subseteq A \hat{\otimes} B \quad L\left(A, B^{*}\right)$. In particular any compact linear operator $T: A \rightarrow B^{*}$ has a decomposition of the form

$$
T=\sum_{n=1}^{\infty} b_{n} \tau^{*} \circ S \circ a_{n} \tau
$$

for some $u=\sum_{n=1}^{\infty} a_{n} \otimes b_{n}$ in $A \hat{\otimes} B$ and some $S$ in $L\left(A, B^{*}\right)$.

(ii) Assume that, for each $a$ in $A, b$ in $B$ and $S$ in $L\left(A, B^{*}\right)$, the operator ${ }_{b} \tau^{*} \circ S \circ{ }_{a} \tau$ is compact. Then the algebra $A \hat{\otimes} B$ is regular iff $L\left(A, B^{*}\right)=K\left(A, B^{*}\right)$.

(iii) Assume that, for each $a$ in $A$ and $b$ in $B$, one of the operators ${ }_{a} \tau$ or ${ }_{b} \tau$ is compact and the other is weakly compact. Then the algebra $A \hat{\otimes} B$ is a right ideal in its second dual; and $A \hat{\otimes} B$ is regular iff $L\left(A, B^{*}\right)=K\left(A, B^{*}\right)$. Moreover, in this case the space $K\left(A, B^{*}\right)$ has the RNP.

Proof. (i) The first inclusion of the assertion is proved in [56; Thm. 4.5]. The second inclusion follows from Theorem 3.1(a) above.

(ii) Assume that, for each $a$ in $A, b$ in $B$ and $S$ in $L\left(A, B^{*}\right)$ the operator ${ }_{b} \tau^{*} \circ S \circ{ }_{a} \tau$ is compact. Let $u=\sum_{n=1}^{\infty} a_{n} \otimes b_{n}$ be a general element of $A \hat{\otimes} B$. Put $S_{n}=\sum_{i=1}^{n} b_{i} \tau^{*} \circ S \circ_{a_{i}} \tau$. The operator $S_{n}$ is compact and as one can see easily, in the operator norm of the space $L\left(A, B^{*}\right), S_{n} \rightarrow{ }_{u} S$ so that ${ }_{u} S$ is compact [14; VI. 5.5]. Whence, $A \hat{\otimes} B L\left(A, B^{*}\right)=K\left(A, B^{*}\right)=\operatorname{wap}(A \hat{\otimes} B)$. From these equalities we conclude that the algebra $A \hat{\otimes} B$ is regular if we have $L\left(A, B^{*}\right)=K\left(A, B^{*}\right)$.

(iii) Assume, for example, that for each $a$ in $A{ }_{a} \tau$ is compact and for each $b$ in $B,{ }_{b} \tau$ is weakly compact. In order to show that the algebra $A \hat{\otimes} B$ is a right ideal in its second dual we shall first show that 
the left multiplication operator $a \otimes b \tau$ on $A \hat{\otimes} B$ is weakly compact. To this end, let $A_{1} \otimes B_{1}$ denote the collection of the elements of the form $a \otimes b$ with $a$ in $A_{1}$ and $b$ in $B_{1}$. By definition of the projective tensor topology (see [28; p. 25]), the unit ball of $A \hat{\otimes} B$ is contained in $\overline{\mathrm{aco}}\left(A_{1} \otimes B_{1}\right)$, the closed absolutely convex hull of $A_{1} \otimes$ $B_{1}$. Therefore the operator $a \otimes b \tau$ is weakly compact iff the set $\left(a A_{1} \otimes\right.$ $b B_{1}$ ) is relatively weakly compact (Krein-Smulian's Theorem [14; V. 6.4]). To prove that this latter set is relatively weakly compact is equivalent to proving that, for any norm convergent sequence $\left(x_{n}\right)$ in $a A_{1}$ with $x_{n} \rightarrow x$, any weakly convergent sequence $\left(y_{n}\right)$ in $b B_{1}$ with $y_{n} \rightarrow y$ weakly and for any $S$ in $L\left(A, B^{*}\right)$, we have $\left\langle S\left(x_{n}\right), y_{n}\right\rangle \rightarrow$ $\langle S(x), y\rangle$. This being immediate to see, we conclude that the operator $a_{a \otimes b} \tau$ is weakly compact. Now, let $u=\sum_{n=1}^{\infty} a_{n} \otimes b_{n}$ be a general element of $A \hat{\otimes} B$. Put $u_{n}=\sum_{i=1}^{n} a_{i} \otimes b_{i}$. Then $u_{n} \tau$ is weakly compact and as one can see very easily, $u_{n} \tau \rightarrow{ }_{u} \tau$ uniformly on the unit ball of $A \hat{\otimes} B$. Hence ${ }_{u} \tau$ is weakly compact [14; VI. 4.4], and $A \hat{\otimes} B$ is a right ideal in its second dual. The last two assertions of (iii) follow, respectively, from part (ii) of this theorem and Corollary 3.7.

As a concrete application of this theorem we give the following corollary, compare with [56; Cor. 4.18].

Corollary 5.4. Let $B$ be a regular Banach algebra with a BLAI. Then the algebra $c_{0} \hat{\otimes} B$ is regular iff $B^{*}$ does not contain a copy of $c_{0}$. Moreover, if $B$ is a right ideal in its second dual then $c_{0} \hat{\otimes} B$ is regular, a right ideal in its second dual and the space $\left(c_{0} \hat{\otimes} B\right)^{*}=K\left(c_{0}, B^{*}\right)=$ $l^{1} \ddot{\otimes} B^{*}$ has the $R N P$.

Proof. We first note that, by Proposition 3.12 and Example 3.14(b), for each $a$ in $c_{0}$, the left multiplication operator ${ }_{a} \tau$ is compact. Therefore, by the preceding theorem, $c_{0} \hat{\otimes} B$ is regular iff $K\left(c_{0}, B^{*}\right)=$ $L\left(c_{0}, B^{*}\right)$. Now remark that any weakly compact operator $T: c_{0} \rightarrow$ $B^{*}$ is compact since $c_{0}^{*}=l^{1}$ has the Schur property. On the other hand, by a result due to A. Pelczynski [40; Thm. 5 and Cor. 1], a linear operator $T: c_{0} \rightarrow B^{*}$ is weakly compact iff $B^{*}$ does not contain a copy of $c_{0}$. From this we conclude that the algebra $c_{0} \hat{\otimes} B$ is regular iff $B^{*}$ does not contain a copy of $c_{0}$. Now, assume that $B$ is a right ideal in its second dual. Then, by Corollary $3.7, B^{*}$ has the RNP so that it does not contain a copy of $c_{0}$. Hence $c_{0} \hat{\otimes} B$ is regular. The rest is immediate from the preceding theorem and Corollary 3.7. 
We end this note with some remarks and questions.

REMARKS AND Questions 5.5. 1. Let $A$ be a Banach algebra with a BLAI. Theorem 3.1(b) says that if $A$ is a right ideal in its second dual then $\operatorname{wap}(A)=A A^{*}$. The converse of this result is not true. Indeed, let $T$ be a locally compact nondiscrete topological space and $A=C_{0}(T)$. Then $\operatorname{wap}(A)=A^{*}=A A^{*}$, but $A$ is not a right ideal in its second dual. On the other hand, if $G$ is a locally compact topological group and $A=L^{1}(G)$ its group algebra, the equality wap $(A)=A A^{*}$ implies that $A$ is a right ideal in its second dual (see Propositions 3.3 and 4.4). This situation raises the following question: Characterize those Banach algebras $A$ for which the equality $\operatorname{wap}(A)=A A^{*}$ implies that $A$ is a right ideal in its second dual.

2. Let $G$ be a locally compact topological group and $A=L^{1}(G)$ be its group algebra. Sakai's theorem [46; Thm. 1] says that if there exists a nonzero element $a$ in $A$ for which ${ }_{a} \tau$ is compact (or weakly compact, see [19; Cor. 3.2]) then $G$ is compact so that $A$ is a right ideal in its second dual. This result suggests the following question: Let $A$ be a Banach algebra with a BLAI and DPP. Does the existence of a nonzero element $a$ in $A$ for which ${ }_{a} \tau$ is compact imply that $A$ is a right ideal in its second dual?

3. In view of Corollary 3.9, the following question suggests itself: Is there a nonreflexive and non-unital WSC regular Banach algebra with a BAI?

4. Let $G$ be a compact group and $A=L^{1}(G)$ its group algebra. As we have seen in $\S 4, \operatorname{wap}(A)=C(G)=\mathrm{WAP}(G)$ and the space $C(G)$ does not contain a copy of $l^{\infty}$. This result suggests the question: Let $G$ be a locally compact group and $w G$ be its weakly almost periodic compactification. Does the space $C(w G)$ contains a copy of $l^{\infty}$ ? Here we remark that $w G$ need not be a topological group.

5. For a Banach algebra $A$, let $s(A)$ denote the collection of the separable subalgebras of $A$ and by $d s(A)$ the subset of $s(A)$ consisting of the subalgebras whose duals are separable. It is immediate from the double limit criterion that $A$ is regular iff any $B$ in $s(A)$ is regular. On the other hand, if $A^{*}$ does not have the RNP, the equivalence " $A$ is regular iff any $B$ in $d s(A)$ is regular" need not be true. For example, let $A=l^{1}(\mathbb{Z})$ be the group algebra of the additive group of integers $\mathbb{Z}$. Then any $B$ in $d s(A)$ is finite dimensional [37; Prop. 2.a.2], so regular but $A$ is not regular. This fact suggests the following question: Characterize those Banach algebras $A$ for which 
the equivalence " $A$ is regular iff every $B$ in $d s(A)$ is regular" holds.

6. In Theorem 3.5 instead of assuming that each set $H_{\alpha}$ has the RNP if we assume that each set $H_{\alpha}$ has the weak RNP (or, say, is conditionally weakly compact) how does this change the conclusion of the theorem?

7. Proposition 3.13 suggests the question: Let $A$ be a semisimple commutative Banach algebra with carrier space $\Phi$. How are the topological properties of $\Phi$ and the operator properties of ${ }_{a} \tau$ 's (resp. geometric properties of $A$ ) connected? For instance, is $\Phi$ dispersed if $A$ does not contain a copy of $l^{1}$ ?

Note added in proof. Professor M. Grosser (Wien) has kindly informed us that the proof of Corollary 3.6 is not complete. It is indeed so and we do not know whether this corollary is valid or not. However even if it turns out to be invalid this will not affect the rest of the paper for we have used this corollary through Proposition 4.5 and this proposition is valid independently from Corollary 3.6 for the following reasons: Any compact topological group is a dyadic space [48; p. 144] and, for a dyadic space $S$, the space (cs) does not contain an isomorphic copy of $l^{\infty}$ [R. Engelkind and A. Pelczynski, Coll. Math. XI (1963), 55-63].

\section{REFERENCES}

[1] C. Akermann, Some mapping properties of the group algebra of a compact group, Pacific J. Math., 22 (1967), 1-8.

[2] R. Arens, The adjoint of a bilinear operation, Proc. Amer. Math. Soc., 2 (1951), 839-848.

[3] J. F. Berglund, H. D. Junghenn and P. Milnes, Compact Right Topological Semigroups and Generalizations of Almost Periodicity, Lecture Notes in Math. No. 663, Springer-Verlag (1978), Berlin.

[4] F. F. Bonsall and J. Duncan, Complete Normed Algebras, Springer-Verlag (1973), New York.

[5] J. Bourgain and G. Pisier, A construction of $L_{\infty}$-space and related Banach spaces, Bol. Soc. Brasil Math., 14 (1983), 109-123.

[6] R. D. Bourgin, Geometric Aspects of Convex Sets with the Radon-Nikodym Property, Lecture Notes in Math. No. 993, Springer-Verlag (1983), New York.

[7] R. B. Burckel, Weakly Almost Periodic Functionals on Semigroups, Gordon and Breach (1970), New York.

[8] Ching Chou, Weak almost periodic functions and almost convergent functions on a group, Trans. Amer. Math. Soc., 206 (1975), 175-200. 
[9] P. Civin, Ideals in the second conjugate of a group algebra, Math. Scand., 11 (1962), 161-174.

[10] P. Civin and B. Yood, The second conjugate space of a Banach algebra as an algebra, Pacific J. Math., 11 (1961), 847-870.

[11] J. Diestel, A survey of results related to the Dunford-Pettis property, Contemporary Math., 2 (1980), 15-60.

[12] J. Diestel and J. J. Ulh, Jr., Vector Measures, Math. Surveys No. 15, Amer. Math. Soc. Providence, R.I. (1977).

[13] J. Duncan and S. A. R. Hosseinium, The second dual of a Banach algebra, Proc. Royal Soc. Edinburgh, 84A (1979), 309-325.

[14] N. Dunford and J. T. Schwartz, Linear Operators I, Wiley (1958), New York.

[15] C. F. Dunkl and D. E. Ramirez, Weakly almost periodic functionals on the Fourier algebra, Trans. Amer. Math. Soc., 185 (1975), 501-514.

[16] H. A. M. Dzinotyiweyi, Nonseparability of quotient spaces of function algebras on topological semigroups, Trans. Amer. Math. Soc., 272 (1982), 223-235.

[17] B. R. Gelbaum, Tensor products of Banach algebras, Canad. J. Math., 11 (1959), 297-310.

[18] F. Ghahramani, Compact elements of weighted group algebras, Pacific J. Math., 113 (1984), 77-84.

[19] __ Weighted group algebra as an ideal in its second dual space, Proc. Amer. Math. Soc., 90 (1984), 71-76.

[20] J. Gil de Lamadrid, Uniform cross norm and tensor products of Banach algebras, Bull. Amer. Math. Soc., 69 (1967), 789-803.

[21] G. Godefroy and B. Iochum, Arens regularity of Banach algebras and the geometry of Banach spaces, J. Funct. Anal., 80 (1988), 47-59.

[22] E. E. Granirer, Exposed points of convex sets and weak sequential convergence, Mem. Amer. Math. Soc. No. 123 (1972).

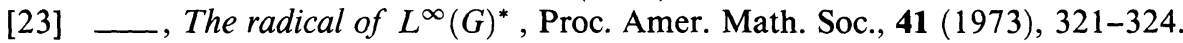

[24] M. Grosser, Arens semi-regular Banach algebras, Monatsh. für Math., 98 (1984), 41-52.

[25] $\_, L^{1}(G)$ as an ideal in its second dual space, Proc. Amer. Math. Soc., 73 (1979), 363-364.

[26] A. Grothendieck, Critères de compacticité dans les espaces fonctionels généraux, Amer. J. Math., 74 (1972), 168-186.

[27] __ Sur les applications lineaires faiblement compactes d'espaces du type $C(K)$, Canad. J. Math., 5 (1953), 129-173.

[28] _ Produits tensoriels topologiques et espaces nucléaires, Mem. Amer. Math. Soc., No. 16 (1955).

[29] S. L. Gulick, The bidual of a locally multiplicatively-convex algebra, Pacific J. Math., 18 (1966), 121-137.

[30] R. Haydon, A non-reflexive Grothendieck space that does not contain $l^{\infty}$, Israel J. Math., 40 (1981), 65-73.

[31] E. Hewitt and K. R. Ross, Abstract Harmonic Analysis 1, Springer-Verlag (1963), Berlin.

[32] __ Abstract Harmonic Analysis 2, Springer-Verlag (1970), Berlin.

[33] N. Issik, J. S. Pym and A. Ülger, The second dual of the group algebra of a compact group, J. London Math. Soc., 35 (1987), 135-148.

[34] R. C. James, Projections in the space $m$, Proc. Amer. Math. Soc., 6 (1955), 899-902.

[35] D. L. Johnson, A characterization of compact groups, Proc. Amer. Math. Soc., 74 (1979), 381-382. 
[36] J. M. Kister, Uniform continuity and compactness in topological groups, Proc. Amer. Math. Soc., 13 (1962), 37-40.

[37] J. Lindenstrauss and L. Tzafriri, Classical Banach Spaces I, Springer-Verlag (1977), Berlin.

[38] T. W. Palmer, The bidual of the compact operators, Trans. Amer. Math. Soc., 288 (1985), 827-840.

[39] A. Pelczynski and Z. Semadeni, Spaces of continuous functions III, Studia Math., 18 (1959), 211-222.

[40] A. Pelczynski, Projections in certain Banach spaces, Studia Math., XIX (1960), 210-228.

[41] J. S. Pym, The convolution of functionals on spaces of bounded functions, Proc. London Math. Soc., 15 (1965), 84-104.

[42] J. S. Pym and A. Ülger, Arens regularity of inductive limits and related matters, Quart. J. Math. Oxford, 40 (1989), 101-109.

[43] M. Rajagopalan, Fourier transform in locally compact groups, Acta Szeged, 25 (1964), 86-89.

[44] H. Reiter, Classical harmonic analysis and locally compact groups, Oxford Math. Monographs, Oxford University Press (1968).

[45] H. R. Rosenthal, Some recent discoveries in the isomorphic theory of Banach spaces, Bull. Amer. Math. Soc., 84 (1978), 803-831.

[46] S. Sakai, Weakly compact operators on operator algebras, Pacific J. Math., 14 (1964), 659-664.

[47] G. L. Seever, Measures on F-spaces, Trans. Amer. Math. Soc., 133 (1968), 267-280.

[48] Z. Semadeni, Banach spaces of continuous functions, Polish Scientific Publishers, Warszawa (1971).

[49] M. Talagrand, Un nouveau $C(K)$ qui possede la propriété de Grothendieck, Israel J. Math., 37 (1980), 181-191.

[50] B. J. Tomiuk and P. K. Wong, The Arens product and duality in $B^{*}$-algebras, Proc. Amer. Math. Soc., 25 (1970), 529-535.

[51] B. J. Tomiuk, Arens regularity and the algebra of double multipliers, Proc. Amer. Math. Soc., 81 (1981), 293-298.

[52] J. Tomiyama, Tensor product of commutative Banach algebras, Tôhuku Math. J., 12 (1960), 147-154.

[53] A. Ülger, Weakly compact bilinear forms and Arens regularity, Proc. Amer. Math. Soc., 101 (1987), 697-704.

[54] _ Arens regularity of the algebra $K(X)$, Monatsh. für Math., 105 (1988), 313-318.

[55] — Continuity of weakly almost periodic functionals on $L^{1}(G)$, Quart. J. Math. (Oxford), 37 (1986), 495-497.

[56] _ Arens regularity of the algebra $A \hat{\otimes} B$, Trans. Amer. Math. Soc., 305 (1988), 623-639.

[57] S. Watanabe, $A$ Banach algebra which is an ideal in the second dual space, Sci. Rep. Niigata Univ. Ser. A, 11 (1974), 95-101.

[58] _ A Banach algebra which is an ideal in the second dual space II, Sci. Rep. Niigata Univ. Ser. A, 13 (1976), 43-48.

[59] J. C. S. Wong, Topologically stationary locally compact groups and amenability, Trans. Amer. Math. Soc., 144 (1969), 351-363.

[60] P. K. Wong, Arens product and the algebra of double multipliers, Proc. Amer. Math. Soc., 94 (1985), 441-443. 
[61] N. J. Young, The irregularity of multiplication in group algebras, Quart. J. Math. (Oxford), 24 (1972), 59-62.

[62] _ Periodicity of functionals and representations of normed algebras on reflexive spaces, Proc. Edinburgh Math. Soc., 20 (1976), 99-120.

Received July 5, 1988 and in revised form March 17, 1989.

BOĞAZIÇI UNIVERSITY

80815 BebeK, Istanbul, TURKey 



\section{PACIFIC JOURNAL OF MATHEMATICS EDITORS}

\author{
V. S. VARADARAJAN \\ (Managing Editor) \\ University of California \\ Los Angeles, CA 90024-1555-05 \\ Herbert Clemens \\ University of Utah \\ Salt Lake City, UT 84112 \\ ThOMAs ENRIGHT \\ University of California, San Diego \\ La Jolla, CA 92093
}

R. FINN

Stanford University

Stanford, CA 94305

HeRmanN FlaschKa

University of Arizona

Tucson, AZ 85721

VAUGHAN F. R. JoNES

University of California

Berkeley, CA 94720

SteVen KerCKhofF

Stanford University

Stanford, CA 94305
ROBION KIRBY

University of California

Berkeley, CA 94720

C. C. Moore

University of California

Berkeley, CA 94720

HAROLD STARK

University of California, San Diego

La Jolla, CA 92093

\section{ASSOCIATE EDITORS}
R. ARENS
E. F. BECKENBACH
B. H. NeumanN
F. Wolf
K. YoshidA
(1906-1982)
(1904-1989)

\section{SUPPORTING INSTITUTIONS}

UNIVERSITY OF ARIZONA
UNIVERSITY OF BRITISH COLUMBIA
CALIFORNIA INSTITUTE OF TECHNOLOGY
UNIVERSITY OF CALIFORNIA
MONTANA STATE UNIVERSITY
UNIVERSITY OF NEVADA, RENO
NEW MEXICO STATE UNIVERSITY
OREGON STATE UNIVERSITY
UNIVERSITY OF ARIZONA
UNIVERSITY OF BRITISH COLUMBIA
UNIVERSITY OF CALIFORNIA
MONTANA STATE UNIVERSITY
NEW MEXICO STATE UNIVERSITY

\author{
UNIVERSITY OF OREGON \\ UNIVERSITY OF SOUTHERN CALIFORNIA \\ STANFORD UNIVERSITY \\ UNIVERSITY OF HAWAII \\ UNIVERSITY OF TOKYO \\ UNIVERSITY OF UTAH \\ WASHINGTON STATE UNIVERSITY \\ UNIVERSITY OF WASHINGTON
}

The Supporting Institutions listed above contribute to the cost of publication of this Journal, but they are not owners or publishers and have no responsibility for its content or policies.

Mathematical papers intended for publication in the Pacific Journal of Mathematics should be in typed form or offset-reproduced (not dittoed), double spaced with large margins. Please do not use built up fractions in the text of the manuscript. However, you may use them in the displayed equations. Underline Greek letters in red, German in green, and script in blue. The first paragraph must be capable of being used separately as a synopsis of the entire paper. In particular it should contain no bibliographic references. Please propose a heading for the odd numbered pages of less than 35 characters. Manuscripts, in triplicate, may be sent to any one of the editors. Please classify according to the 1980 Mathematics Subject Classification (1985 Revision) scheme which can be found in the December index volumes of Mathematical Reviews. Supply name and address of author to whom proofs should be sent. All other communications should be addressed to the managing editor, or Elaine Barth, University of California, Los Angeles, California 90024-1555-05.

There are page-charges associated with articles appearing in the Pacific Journal of Mathematics. These charges are expected to be paid by the author's University, Government Agency or Company. If the author or authors do not have access to such Institutional support these charges are waived. Single authors will receive 50 free reprints; joint authors will receive a total of 100 free reprints. Additional copies may be obtained at cost in multiples of 50 .

The Pacific Journal of Mathematics is issued monthly as of January 1966. Regular subscription rate: $\$ 190.00$ a year (6 Vols., 12 issues). Special rate: $\$ 95.00$ a year to individual members of supporting institutions.

Subscriptions, orders for numbers issued in the last three calendar years, and changes of address should be sent to Pacific Journal of Mathematics, P.O. Box 969, Carmel Valley, CA 93924, U.S.A. Old back numbers obtainable from Kraus Periodicals Co., Route 100, Millwood, NY 10546.

The Pacific Journal of Mathematics at P.O. Box 969, Carmel Valley, CA 93924 (ISSN 0030-8730) is published monthly. Second-class postage paid at Carmel Valley, California 93924, and additional mailing offices. Postmaster: send address changes to Pacific Journal of Mathematics, P.O. Box 969, Carmel Valley, CA 93924.

\section{PUBLISHED BY PACIFIC JOURNAL OF MATHEMATICS, A NON-PROFIT CORPORATION}




\section{Pacific Journal of Mathematics}

\section{Vol. 143, No. $2 \quad$ April, 1990}

Gustavo Corach, Horacio Porta and Lázaro Recht, Differential geometry of systems of projections in Banach algebras ................. 209

Peter Fleischmann and Jens Carsten Jantzen, Simple periodic modules of

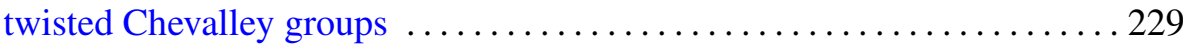

Niels Gronbaek, Amenability of discrete convolution algebras, the commutative case ...................................243

Nguyên H. V. Hung, The mod 2 equivariant cohomology algebras of

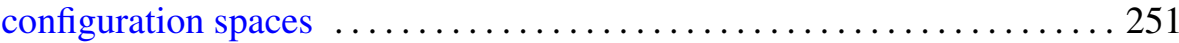

Wojciech Kucharz, Global almost analytic algebraicity of analytic sets . . . 287 John Merrill, A class of consistent anti-Martin's axioms .............. 301

Takafumi Murai, The power 3/2 appearing in the estimate of analytic

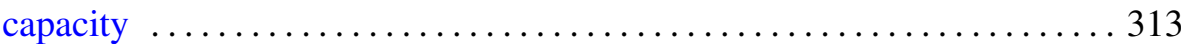

L. Panaitopol and Doru Stefanescu, On the generalized difference

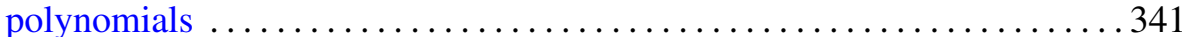

Katsuhiro Shiohama, Takashi Shioya and Minoru Tanaka, Mass of rays

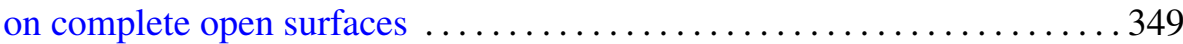

Gerhard Ströhmer, About compressible viscous fluid flow in a bounded

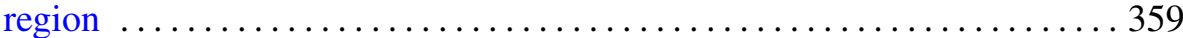

A. Ülger, Arens regularity sometimes implies the RNP 